\section{POS0881 DETECTION OF THE GENE EXPRESSIONS IN PERIPHERAL BLOOD INVOLVED IN THE PROGRESSION OF PULMONARY VESSEL DISEASE AT THE SUBCLINICAL STAGE OF PULMONARY HYPERTENSION ASSOCIATED WITH SYSTEMIC SCLEROSIS}

Y. Koyama ${ }^{1}$, Y. Sato ${ }^{2}$, T. Shoji ${ }^{2}$, S. Fuke ${ }^{3}$, T. Umayahara ${ }^{4}$, M. Sakamoto ${ }^{1}$ ${ }^{1}$ Japanese Red Cross Okayama Hospital, Rheumatology, Center for Autoimmune Diseases, Okayama, Japan; ${ }^{2}$ DNA Chip Research Inc., N/A, Tokyo, Japan; ${ }^{3}$ Japanese Red Cross Okayama Hospital, Cardiology, Okayama, Japan; ${ }^{4}$ Japanese Red Cross Okayama Hospital, Dermatology, Okayama, Japan

Background: Pulmonary hypertension $(\mathrm{PH})$ is prominent as a vascular involvement of systemic sclerosis (SSc), which remains a leading cause of death in spite of current best treatments. Recently, hemodynamic definition of $\mathrm{PH}$ was updated from $m P A P \geq 25 \mathrm{mmHg}$ to $\mathrm{mPAP}>20 \mathrm{mmHg}$ and $P V R \geq 3 W U$. Although new definition may improve the prognosis of $\mathrm{PH}$ associated with $\mathrm{SSc}$ by giving a chance to start management early, it may be insufficient as more than $2 / 3$ of the pulmonary circulation is already impaired by the time of meeting the definition. Therefore, the ideal therapeutic intervention should be started at the subclinical stage of PH in SSc patients, but little is known about underlying pathological mechanisms at the stage. In this study, we investigate progression to exercise-induced $\mathrm{PH}(\mathrm{exPH})^{1)}$, which is considered subclinical $\mathrm{PH}$, in the prospective registry of high-risk population for developing $\mathrm{PH}$ associated with SSc.

Objectives: To detect the gene expressions in peripheral blood involved in the progression of pulmonary vessel disease (PVD) at the subclinical stage of $\mathrm{PH}$ associated with SSc.

Methods: Total of 180 patients who had not met PH criteria with Raynaud phenomenon, skin sclerosis or SSc-related autoantibody was registered. To detect the early PVD, exercise Doppler echocardiography (exDE) was carried out every 6 or 12 months for up to 6 years. The definition of exPH was maximum sPAP $>40 \mathrm{mmHg}$ or increase in sPAP $>20 \mathrm{mmHg}$ estimated by exDE during exercise. For gene expression analysis, total RNAs from whole peripheral blood cells were extracted by PAXgene system, and then multiplex sequencing was done. To identify candidate genes involved in the progression to exPH, random forest machine learning method was employed. Volcano plots, a scatter plots to visualize fold-changes and p-values of differentially expressed genes (DEGs) between exPH and others (exN), were also used for seeking the important genes for disease progression.

Results: At the time of registration, $34.4 \%$ of patients met exPH criteria, and $15.6 \%$ of patients developed exPH during follow-up period ( $35.0 \pm 18.1$ months). Expression of TNF gene was selected as the most useful genes to predict progression to exPH by random forest, and the accuracy of the model was about $87 \%$. Volcano plots indicated that expressions of TMEM176A and TMEM176B were prominent (fold-change $>2.4$ and $-\log 10 \mathrm{p}$-value $>3.5$ ) in exPH patients. The accuracy was improved to $90 \%$ if the expression of TNF and TMEMA/B were used for the prediction of progression to exPH. We found that statistically significant increase in the expression of TNF was eliminated at the time of fulfilling the exPH criteria, while increase in expressions of TMEM A/B were still kept.

Conclusion: It was reported that TNF $\alpha$ drives pulmonary arterial hypertension by suppressing the BMP type-II receptor and altering NOTCH signalling ${ }^{2}$. Our findings suggest that TNF $\alpha$ plays important role only in the period of pre-exPH. On the other hand, increase in expressions of TMEM A/B were observed through the period of pre-exPH to post-exPH. It suggests that there are multiple phases before developing $\mathrm{PH}$ associated with SSc. It is very important to understand the phases for the precise treatment to arrest the progression of PVD. REFERENCES

[1] R. Naeije et al., Am J resp and critical care med 187, 576-583 (2013). 2) LA. Hurst et al., Nat Commun. 13;8:14079 (2017).

Disclosure of Interests: Yoshinobu Koyama Speakers bureau: Asahikasei, Ayumi, BMS, Mitsubishi Tanabe, Shin-nihon, Paid instructor for: Asahikasei, Asteras, BMS, Grant/research support from: Eli-Lilly, Yoshiharu Sato: None declared, Tatsuma Shoji: None declared, Soichiro Fuke: None declared, Takatsune Umayahara: None declared, Moe Sakamoto: None declared DOI: 10.1136/annrheumdis-2021-eular.3609

\section{POS0882 AMINAPHTONE LONG-TERM TREATMENT IN SYSTEMIC SCLEROSIS}

A. Sulli ${ }^{1}$, E. Gotelli ${ }^{1}$, A. Cere ${ }^{1}$, E. Hysa ${ }^{1}$, G. Pacini ${ }^{1}$, C. Pizzorni ${ }^{1}$, S. Paolino ${ }^{1}$, M. Cutolo ${ }^{1}{ }^{1}$ Laboratory of Experimental Rheumatology and Academic Division of Clinical Rheumatology, Department of Internal Medicine, University of Genova, IRCCS San Martino Polyclinic Hospital, Genova, Italy

Background: Aminaphtone has been used since many years to treat microvascular disorders. Aminaphtone seems to improve clinical symptoms of Raynaud's phenomenon (RP), either primary or secondary to systemic sclerosis (SSc) by increasing peripheral blood perfusion as assessed by Laser speckle contrast analysis (1).

Objectives: To evaluate long-term survival and tolerability of Aminaphtone in SSc patients with secondary RP.

Methods: Eighty SSc patients (mean age 64 \pm 12 years; mean disease duration $9 \pm 8$ years) treated with Aminaphtone due to active RP were enrolled (ACR EULAR 2013 criteria). Patients were taking also various concomitant treatments, including aspirin, cyclic intravenous iloprost, immunomodulators, endothelin receptor antagonists. SSc patients performed periodic clinical and laboratory assessments on average every four months per our clinical practice. Duration of Aminaphtone administration, side effects, and self-assessment of Raynaud Condition Score (RCS) with a scale from 0 (absence of pain) to 10 (maximal pain) were retrospectively assessed.

Results: The observation period was between twelve and seventy months (mean $36 \pm 19$ months). Aminaphtone was administered at $75 \mathrm{mg}$ twice daily, as standard initial posology per our clinical practice. During the follow-up, five patients $(6.2 \%)$ referred headache as side effect: three of them had to reduce Aminaphtone posology to $75 \mathrm{mg}$ per day, while maintaining clinical benefits; two patients had to stop the treatment. No other side effects related to the drug appeared during the treatment period, and repeated blood tests did not reveal any significant alteration ascribable to Aminaphtone. After 3 months of treatment sixty-six patients $(83 \%)$ referred a subjective improvement of RP (RCS $3.6 \pm 0.8$, vs baseline RCS $7.4 \pm 0.8, p=0.032)$, whereas fourteen patients $(17 \%)$ were clinically unsatisfied (RCS 6.1 $\pm 0.4, p=0.12$ ). In this last group of patients, Aminaphtone posology was increased to $75 \mathrm{mg}$ three times a day with a satisfactory amelioration in furthe nine patients $(94 \%$ of total) (RCS $4.0 \pm 0.6, p=0.04$ ), while five patients $(6.2 \%)$ definitively discontinued therapy for subjective ineffectiveness within six months Patients referred a sustained improvement of RCS along the observational period ( $36 \pm 19$ months) (last RCS $3.6 \pm 0.7$ vs baseline, $p=0.031$ ).

Conclusion: During an average observation period of three years, Aminaphtone showed a good tolerability profile along with sustained efficacy in $94 \%$ of patients with SSc-related RP, without disabling side effects. The absence of a placebo-control group, the retrospective design limit the results, and a randomized controlled trial for Aminaphtone use in the management of SSc-related RP is needed.

\section{REFERENCES:}

[1] Ruaro B et al. 2019. Front Pharmacol 10:293.

Disclosure of Interests: Alberto Sulli: None declared, Emanuele Gotelli: None declared, ANDREA CERE: None declared, Elvis Hysa: None declared, Greta Pacini: None declared, Carmen Pizzorni: None declared, Sabrina Paolino: None declared, Maurizio Cutolo Grant/research support from: Laboratori Baldacci s.p.a

DOI: 10.1136/annrheumdis-2021-eular.3614

\section{POS0883 \\ DETECTION OF AUTOANTIBODIES AGAINST MUSCLE- SPECIFIC FOUR-AND-A-HALF-LIM DOMAIN 1 (FHL1) IN INFLAMMATORY MYOPATHIES: RESULTS FROM A SINGLE-CENTER COHORT}

A. S. Galindo-Feria ${ }^{1,2}$, B. Horuluoglu ${ }^{1,2}$, J. Day $^{3}$, C. Cerqueira ${ }^{1,2}$, S. Proudman ${ }^{3}$, I. E. Lundberg ${ }^{1,2}$, V. Limaye ${ }^{3} .{ }^{1}$ Karolinska Institutet, Solna, Karolinska University Hospital, Division of Rheumatology, Department of Medicine, Stockholm, Sweden; ${ }^{2}$ Karolinska Institutet and Karolinska University Hospital Solna, Center for Molecular Medicine, Stockholm, Sweden; ${ }^{3}$ Royal Adelaide Hospital, University of Adelaide, Rheumatology Unit and Discipline of Medicine, Adelaide, Australia

Background: Autoantibodies targeting a muscle-specific autoantigen, four-anda-half-LIM-domain 1 (FHL1), have been previously identified in patients with idiopathic inflammatory myopathies (IIM) (1).

Objectives: The aim of this project was to determine the prevalence and associations of anti-FHL antibody in South Australian patients with histologically-confirmed IIM and in an autoimmune disease control (systemic sclerosis (SSc)). Methods: Sera from patients with IIM $(n=267)$ from the South Australian Myositis Database (SAMD), and SSc $(n=174)$ from the Australian Scleroderma Cohort Study (ASCS) followed at the Royal Adelaide Hospital, and healthy controls (HC, $\mathrm{n}=100$ ) were analyzed for anti-FHL1 autoantibodies by Enzyme-Linked ImmunoSorbent Assay (ELISA). Clinical, serological and histological details were retrieved from the SAMD and the ASCS.

Results: Autoantibodies to FHL1 were more frequent in patients with IIM $(55 / 267,20.5 \%)$ compared with SSc $(18 / 174,10 \%)(p<0.001)$ and HC $(4 / 100,4 \%)$ $(p<0.001)$. Muscular vessel inflammation and atrophy were seen more frequently in IIM anti-FHL1 ${ }^{+}$patients compared with anti-FHL1 ${ }^{-}(p<0.01$ and $p<0.05)$. Dysphagia, marked muscle atrophy, and high $\mathrm{CK}$ levels were frequent in anti-FHL1 ${ }^{+}$ patients with inclusion body myositis (IBM) and immune-mediated necrotizing myopathy (IMNM). In 35/54 anti-FHL1+ patients, there were no other myositis-specific autoantibodies present. Anti-FHL1 autoantibodies in patients with $\mathrm{SSc}$ were associated with gastric antral vascular ectasia. 
Conclusion: Anti-FHL1 autoantibodies were detected in $20.5 \%$ of IIM patients. In IBM and IMNM, the presence of anti-FHL1-autoantibodies was associated with a severe myopathy as suggested by presence of dysphagia and muscle atrophy.

REFERENCES:

[1] Albrecht I, Wick C, Hallgren A, Tjarnlund A, Nagaraju K, Andrade F, et al. Development of autoantibodies against muscle-specific FHL1 in severe inflammatory myopathies. J Clin Invest. 2015;125(12):4612-24.

Disclosure of Interests: Angeles Shunashy Galindo-Feria: None declared, Begum Horuluoglu: None declared, Jessica Day: None declared, Catia Cerqueira: None declared, Susanna Proudman: None declared, Ingrid E. Lundberg Consultant of: Consulting fees from Corbus Pharmaceuticals, Inc, Grant/research support from: Research grants from Bristol Myers Squibb and Astra Zeneca, Vidya Limaye Consultant of: Scientific adviser for Actelion and Boehringer-Ingelheim, Grant/research support from: PI for clinical trials for Bayer, Boehringer-Ingelheim, Corbus, and CSL

DOI: 10.1136/annrheumdis-2021-eular.3640

\section{POS0884 \\ THE ENHANCED LIVER FIBROSIS (ELF) SCORE AS A BIOMARKER OF SKIN FIBROSIS IN SYSTEMIC SCLEROSIS}

C. Chen ${ }^{1}$, S. Yang ${ }^{1}$, Z. Jiang ${ }^{1}$, W. Wann ${ }^{1}$, H. Zou' ${ }^{1}$ M. Liang ${ }^{1} .{ }^{1}$ Huashan Hospital Affiliated to Fudan University, Department of Rheumatology, Shanghai, China

Background: Serum fibrotic markers for systemic sclerosis (SSc) remain limited. The Enhanced Liver Fibrosis (ELF) score, originally derived and validated in patients with chronic liver disease, is an algorithm combining 3 serum markers, known as procollagen type III amino terminal propeptide (PIIINP), tissue inhibitor of metalloproteinases 1 (TIMP-1), and hyaluronic acid (HA). The combined score was proved to be superior to the single components in reflecting the severity of liver fibrosis. However, the performance of ELF score and its components has not been fully validated in SSc.

Objectives: To investigate PIIINP, TIMP-1, HA, and the combined algorithm ELF score as fibrotic markers for SSc skin involvement.

Methods: Eighty SSc patients (44 dcSSc and 36 IcSSc), fulfilling the 2013 ACR/EULAR criteria with the absence of chronic liver diseases, were enrolled. Eighty age- and sex- matched healthy controls were also included. Serum PIIINP and HA levels were quantified by chemiluminescence immunoassay. Serum TIMP-1 levels were determined by enzyme-linked immunosorbent assay. The ELF score was calculated using the formula ELF score= $2.494+0.846^{*} \ln (H A)+0.735^{*} \ln (P I I I N P)+0.391^{*} \ln (T I M P-1)$. Results were correlated with clinical profiles including modified Rodnan skin score (mRSS) and interstitial lung disease (ILD).

Results: Compared with healthy controls, patients with SSc showed significantly elevated serum PIIINP (11.2 \pm 4.8 vs. $5.73 \pm 1.4 \mu \mathrm{g} / \mathrm{L}, \mathrm{p}<0.001)$, TIMP-I $(123.7 \pm 78.6$ vs. $67.8 \pm 26.5 \mathrm{ng} / \mathrm{ml}, \mathrm{p}<0.001)$, and ELF score $(10.5 \pm 0.9$ vs. $9.7 \pm 0.4, P<0.001)$. Even higher levels of PIIINP, TIMP-1, and ELF score were observed in dcSSc patients, compared with IcSSc patients $(p<0.001, p=0.024$, $p=0.003$, respectively). No significant difference was found in the levels of serum HA between patients and controls. Strong correlations were observed between mRSS and ELF score $(r=0.54, p<0.001)$, and between $m R S S$ and PIIINP $(r=0.62, p<0.001)$, whereas only weak correlations could be observed between mRSS and TIMP-1 $(r=0.28, p=0.02)$, and between mRSS and HA $(r=0.26, p=0.03)$. When stratified by ELF score, using cutoffs proposed for liver fibrosis and cirrhosis, SSc patients with ELF $<9.8$ showed the lowest mRSS on average, while patients with ELF>11.3 showed the highest $(p<0.001)$. When stratified by serum PIIINP levels, using the 25th and 75th percentiles, SSc patients with serum PIIIINP levels $<7.8 \mu \mathrm{g} / \mathrm{L}$ showed the lowest mRSS on average, while patients with PIIINP $>14.0 \mu \mathrm{g} / \mathrm{L}$ showed the highest $(\mathrm{p}<0.001)$. Neither the ELF score nor its components showed significant difference between patients with and without ILD.

Conclusion: The ELF score could be used for reflecting the severity of overall skin involvement in SSc, and serum PIIINP also increased in parallel with the increase of mRSS. Longitudinal prospective studies exploring ELF score or serum PIIINP as fibrotic markers and outcome measures of SSc are warranted.

\section{REFERENCES}

[1] Lichtinghagen R, Pietsch D, Bantel H, et al. The Enhanced Liver Fibrosis (ELF) score: Normal values, influence factors and proposed cut-off values. Journal of Hepatology. 2013; 59: 236-42.

[2] Abignano G, Blagojevic J, Bissell LA, et al. European multicentre study validates enhanced liver fibrosis test as biomarker of fibrosis in systemic sclerosis. Rheumatology. 2019; 58: 254-59.
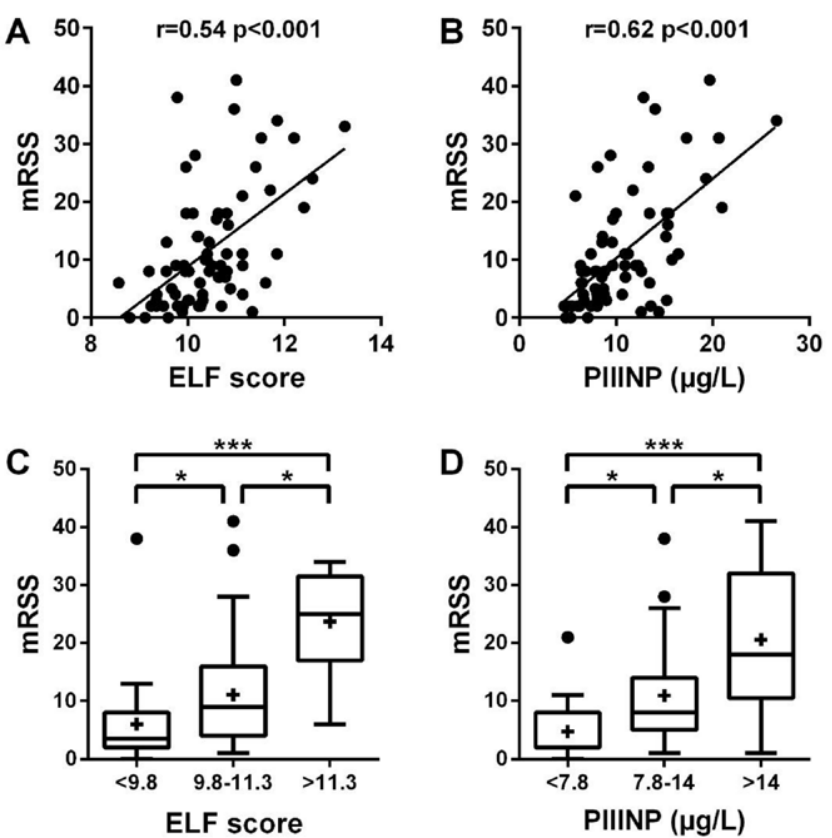

Box and whisker plots: lines from bottom to top indicated the minimum, $25^{\text {th }}$ percentile, median, $75^{\text {th }}$ percentile, and the maximum, outliers were identified by Turkey's method. Statistical analysis: (AB) Pearson's correlation and $(C D)$ Kruskal-Wallis test with Dunn's correction for multiple comparisons. + mean • outlier ${ }^{*} p<0.05{ }^{* *} p<0.01{ }^{* * *} p<0.001$

Figure 1. Correlations of mRSS with ELF score $(A)$ and serum PIIINP $(B)$ and distribution of mRSS among different ELF $(C)$ and PIIINP $(D)$ ranges.

Acknowledgements: The authors have no acknowledgements to declare Disclosure of Interests: None declared

DOI: 10.1136/annrheumdis-2021-eular.3648

\begin{tabular}{l|l}
\hline POS0885 & HIGH INCIDENCE AND MORTALITY OF \\
& PNEUMOCYSTIS JIROVECI INFECTION IN ANTI- \\
& MDA5-ANTIBODY POSITIVE DERMATOMYOSITIS: \\
& EXPERIENCE FROM A SINGLE CENTER
\end{tabular}

Q. Yan ${ }^{1}$, S. Chen ${ }^{1}$, L. Huang ${ }^{1}$, Q. Fu ${ }^{1}$, Y. Ye ${ }^{1} .{ }^{1}$ Renji Hospital, Shanghai Jiaotong University, School of Medicine, Department of Rheumatology, Shanghai, China

Background: Idiopathic inflammatory myopathies (IIM) was associated with a significantly higher risk of opportunistic infections that including Pneumocystis jiroveci pneumonia(PJP) which is potentially fatal opportunistic infection. However, no prior studies have evaluated the PJP infection in subtypes of IIM.

Objectives: To investigate the incidence rate and mortality rate of PJP infection in subgroups of IIM patients according to myopathy specific antibodies.

Methods: In the first part, we reviewed 463 consecutive patients with IIM retrospectively to analyze incidence of PJP infection. In the next part, we enrolled 30 consecutive PJP infection patients with any rheumatic disease was to identify the mortality rate and risk factors. Kaplan-Meier curve with log rank test was used to access differences in survival. Univariate and multivariate analyses were performed to identify prognostic factors using Cox regression.

Results: We found that 12(7.5\%) PJP cases occurred in 160 anti-MDA5-ab-positive DM patients, while only two $(0.7 \%)$ PJP cases were found in 303 anti-MDA5ab-negtive $D M / P M$ patients $(P<0.05)$. PJP infection typically happened in the first two months of the treatment for anti-MDA5-ab-positive DM patients who have a significant decrease in the CD4+ T cell counts and Lymphocyte counts ( $P$ $<0.05)$. Only two (16.7\%) anti-MDA5-ab-positive DM patients recover from PJP with lethally higher mortality than those PJP infection with other rheumatic diseases $(83.3 \%$ vs. $38.9 \%, P<0.05)$. We found no association between the time to anti-PJP treatment and treatment outcomes in anti-MDA5-ab-positive DM; ye we confirmed in PJP infection with other rheumatic diseases that anti-PJP treatment within 6 days crucially increased the survival $(P<0.05)$

Conclusion: PJP infection has alarming high incidence and mortality in antiMDA5-ab-positive DM patients. Unlike PJP infection with other rheumatic diseases, timely treatment for PJP doesn't improve the prognosis of this particular subtype. Therefore, the necessity of further study of PJP prophylaxis treatment in anti-MDA5-ab-positive DM patients is verified. 